\title{
Predictive Value of Interleukin-6 (IL6) in Term Neonates With Early Sepsis During 2010-2011
}

\author{
Marzie Heidarzadeh Arani ${ }^{1}$, Amirhosein Movahedian ${ }^{1}$, Mohammad Ghannaee Arani ${ }^{2}$
}

\author{
Mojtaba Adinah ${ }^{1}$, Ziba Mosayebi ${ }^{3, *}$ \\ ${ }^{1}$ Department of Pediatrics and Neonatology, School of Medicine, Kashan University of Medical Sciences, Kashan, IR Iran \\ ${ }^{2}$ Trauma Research Centre, Kashan University of Medical Sciences, Kashan, IR Iran \\ 3 Department of Pediatrics and Neonatology, School of Medicine, Tehran University of Medical Sciences, Tehran, IR Iran \\ ${ }^{*}$ Corresponding author: Ziba Mosayebi, Department of Pediatrics and Neonatology, School of Medicine, Tehran University of Medical Sciences, Tehran, IR Iran. Tel: +98-3615550021, \\ Fax:+98-3615550021, E-mail: mohammadarani@yahoo.com.
}

Received: October 21, 2012; Revised: January 11, 2013; Accepted: March 9, 2013

\begin{abstract}
Background: Neonatal sepsis is one of the main causes of neonatal mortality and morbidity. It breaks out in one to ten cases per 1000 live births with a mortality rate of 10 to 50 percent. However, the rate of hospital admission for neonates suspicious to systemic infections is more than that for neonates with sepsis which is due to the nonspecific signs and symptoms in the neonatal sepsis.

Objectives: This study was conducted to measure interleukin 6 (IL-6) plasma level as a sign for early detection of neonatal infection.

Materials and Methods: The level of plasma IL-6 and blood culture were checked in all the term neonates suspicious to sepsis admitted to the neonatal intensive care unit of Shahid Beheshti hospital of Kashan during 2010-2011. Positive blood culture was considered as definite sepsis and negative blood culture as suspicious sepsis. Then, the level of IL-6 was compared with the state of blood culture. Premature neonates with low APGAR score, ages more than seven days old, and history of previous admission were excluded from the study due to the probable effect on plasma IL-6 level.

Results: Seventy four cases were male and 68 were female. The prevalence of early neonatal sepsis was reported to be $7 \%$. The etiologic bacterial agents were streptococcus in Group B (GBS) and staphylococcus Epidermis (SE). The most common symptom observable in the patients was respiratory distress (38.6\%). The average level of IL-6 was $1545.65 \mathrm{pg} / \mathrm{mL}$ for the first group (admitted with sepsis signs and symptoms and positive blood culture) and $14.79 \mathrm{pg} / \mathrm{mL}$ for the second one (admitted with sepsis signs and symptoms and negative blood culture), while in the control group, it was $11.04 \mathrm{pg} / \mathrm{mL}$.

Conclusions: A comparative test indicates that there is a significant difference between the levels of interleukin- 6 in three groups (Pvalue $=0.001$ ). This result, not only omits all the probable confounding factors in plasma level of IL-6, but also emphasizes the role of this useful inflammatory marker in detection of neonatal sepsis.
\end{abstract}

Keywords: Early Sepsis; Interleukin-6; Term Neonates

\section{Background}

Neonatal sepsis is a generalized bacterial infection in the blood. It is found in neonates during the first month of life which has been known as a major cause of mortality and morbidity in the newborns. The prevalence of neonatal sepsis has been reported to be different in various countries. Neonatal sepsis occurs in one to four cases per 1000 live births in the developed countries while in many developing countries, it has been 10 times greater than that in the developed countries (1-4). Despite medical advances in healthcare and decreasing sepsis-related mortality (from 90\% to 10-50\%), the diagnosis of sepsis is still considered as one of the major problems for detecting the diseases in infants and children since the clinical symptoms and laboratory findings of neonatal sepsis are often non-specific and these symptoms can also happen in other non-infectious neonatal diseases such as aspiration syndrome, and gastrointestinal blockages (1-5).

Today, laboratory tests for the diagnosis of neonatal sepsis have not shown sufficient specificity. Moreover, these tests take more time; the blood culture as the "gold standard" in the diagnosis of neonatal sepsis is ready two to three days after the sampling (the time is needed for the growth or lack of micro-organism growth causing infections) (1-3). However, if we retrospectively study infants who have been under investigation and treatment of neonatal sepsis with clinical symptoms suspected to early infection during the first week of life, we notice that most of them don't need antibiotic because only less than 10 percent of these neonates (regarding the incidence of early neonatal sepsis) had definitive neonatal sepsis and about 90 percent of them have received it (3).

Implication for health policy/practice/research/medical education:

Since neonatal sepsis is one of the main causes of neonatal mortality and morbidity, measuring interleukin 6(IL-6) plasma level as a sign for early detection of neonatal infection can be useful.

Copyright @ 2013, Ahvaz JundishapurUniversity of Medical Sciences; Published by Kowsar Corp. This is an open-access article distributed under the terms of the Creative Commons Attribution License, which permits unrestricted use, distribution, and reproduction in any medium, provided the original work is properly cited. 
A major effort was made to evaluate and find a criterion that can quickly recognize neonatal sepsis since 1990 . Findings of various studies are considerable in this regard. Different laboratory criteria such as CRP, WBC, immature PMN/mature PMN, blood culture and clinical criteria such as number of primary symptoms of neonates suspicious to neonatal sepsis, and comparison of the primary symptoms have all been used in this respect, but the their certainty was not confirmed (3). In recent years, most studies and reviews have confirmed the role of inflammatory factors such as cytokines and Interleukins - 6 and -8 in neonatal infection because these markers are released immediately at the beginning of neonatal infection from the liver and immune cells and then increased their blood levels. This inflammatory marker has a vital role in most of the inflammatory processes and immunity of the body against viral and bacterial infections so that it functions as a phagocytotic agent for bacteria and viruses $(1,4-6)$. Although these levels decrease within 1224 hours, these inflammatory markers are inducers in production and release of other markers such as CRP and chemotaxy of immune cells to fight infection (1-3).

\section{Objectives}

On the whole, this study focused on the significance of sepsis in neonates and also emphasized the importance of recognizing the inflammatory marker (IL-6) as a diagnostic sign for early neonatal sepsis.

\section{Materials and Methods}

This descriptive analytical study was performed on all term neonates admitted to neonatal intensive care unit in Shahid Beheshti Hospital of Kashan during 2010-2011. The neonates were admitted to the hospital because of the suspected sepsis, their diagnostic tests were sent to the laboratory and antibiotic treatment was then started. Since the aim of this study was to diagnose the early neonatal sepsis, the neonates who had been admitted for longer than seven days were excluded from the study. Signs and symptoms of the suspected sepsis included respiratory symptoms (tachypnea, apnea, respiratory distress, respiratory retraction), digestive symptoms (vomiting, poor feeding, abdominal distention), cardiovascular symptoms (cyanosis, hypotension, weak pulse rate), neurological symptoms (seizure, lethargy, poor neonatal reflexes), and cutaneous symptoms (fever, hypothermia, skin molting).

Moreover, considering previous studies which have been done to evaluate the level of IL-6, neonates with potential confounding factors in their plasma levels of IL-6 such as clearly apparent anomaly (malformation), congenital heart anomalies, prematurity, birth APGAR score less than seven (probability of birth asphyxia) and admission in the previous seven days were excluded from the study. In each admitted neonate, the routine neonatal samples (complete blood count, distinguishing characteristics of blood cells, electrolytes, and the levels of arterial blood gases) were taken to diagnose the sepsis infection.

At first, the blood cultures of neonates were taken from the neonates and in the Bactek neonatal blood culture medium, Becton Dickinson device (Beckton Dickinson, USA) was used as a gold test for the diagnosis of the definitive neonatal sepsis then, about $2 \mathrm{ml}$ blood samples of these neonates were centrifuged, their blood plasma was immediately frozen at $-20^{\circ} \mathrm{C}$ and quickly submitted to the reference. The level of IL- 6 in ten-fold samples was measured using electrochemiluminescence technique or Elecsys 2010 (Roche-Hitachi, Germany) within 2-10 hours which then were recorded in the table of statistical data. This measurement which was made automatically by the machine and laboratory technicians did not interfere with the process.

These neonates, as routine care procedures of neonatal intensive care unit of Kashan Shahid Beheshti Hospital, received the antibiotic and the supportive treatment; considering their general conditions and laboratory findings, additional measurements such as the respiratory assistance with mechanical ventilation, adding antibiotic diet, imaging interventions and other required measures were done on them. If the neonate's general condition was good and his blood culture was also negative, the antibiotic treatment would stop after three days and the neonate would be transferred to the neonatal ward. The plasma level of IL-6 was checked by Elecsys 2010 (Roche-Hitachi) within 12 hours after the neonate's admission. Sampling had been continued until the results of test done on 10 samples were positive regarding their bacterial blood culture in the neonates with suspected neonatal infection. Bactec blood cultures of a total of 132 neonates were obtained. Regarding the normal level of IL-6 in healthy neonates, it had been checked in ten healthy and term neonates with the same age and weight who had been admitted for severe physiologic jaundice to the positive blood culture group.

Finally, the neonates were classified into three groups:

Group 1) the admitted neonates with positive blood cultures suspected to neonatal sepsis

Group 2) the admitted neonates with negative blood cultures suspected to neonatal sepsis

Group 3) the healthy and term neonates with similar age and weight who were admitted for the severe physiologic jaundice to the group with positive blood cultures (control group)Information of pregnant mothers (pregnancy data, the previous histories of disease or admission, the history of drug use, type of delivery, histories of abortion or stillbirth, gestational age and the rupture of the fetal membranes, information of neonates (the reasons of admission, time of admission, gender, age, history of previous admission, blood culture status, and the level of IL-6) were collected and then analyzed using 
Heidarzadeh Arani M et al.

SPSS software and descriptive statistics. Also, ANOVA was used to compare the levels of IL- 6 in the above-mentioned three groups.

\section{Results}

Our study cases included 142 term neonates of which $74(52 / 1 \%)$ were male and the others were female with a mean age of $38.2 \pm 0.86$ (37-41 weeks old). In the positive blood culture group, the most common micro-organism detected were Group B Streptococci (GBS) and Staphylococcus epidermidis , and three cases were affected by these micro-organisms (Table 1).The most common clinical feature was respiratory distress (51 (38.6\%) cases) (Table 2 ).

The average level of IL- 6 in the first group (admitted with

Table 1. Frequency of Occurrence of Microorganisms Isolated From Blood Cultures

\begin{tabular}{ll}
\hline Blood Culture-Microorganism & Frequency, No. , \% \\
\hline Negative & $(93) 132$ \\
\hline Group B Streptococci (GBS) & $3(2.1)$ \\
\hline Staphylococcus epidermidis & $3(2.1)$ \\
\hline Alpha hemolytic Staphylococcus & $1(0.7)$ \\
\hline Non hemolytic Streptococci & $1(0.7)$ \\
\hline Geram positive basilli & $1(0.7)$ \\
\hline Escherichia coli & $1(0.7)$ \\
\hline Total & $(100) 142$ \\
\hline
\end{tabular}

${ }^{\mathrm{a}}$ In group A, the most common bacteria identified in Bactec blood cultures were Group B Streptococci (GBS) and
Table 2. Frequency of Clinical Sign in Neonates Admitted for Neonatal Sepsis

\begin{tabular}{ll}
\hline Clinical Sign & Frequency, No., \% ${ }^{\mathrm{a}}$ \\
\hline Respiratory Distress & $51(38.6)$ \\
\hline Fever & $18(13.6)$ \\
\hline Poor feeding & $30(22.7)$ \\
\hline Recurrent Vomiting & $9(6.8)$ \\
\hline Seizure & $3(2.3)$ \\
\hline Cyanosis & $6(4.5)$ \\
\hline Skin lesion & $4(3)$ \\
\hline Abdominal Distention & $2(1.5)$ \\
\hline Irritability & $1(0.8)$ \\
\hline Hypotonia & $1(0.8)$ \\
\hline Hyporeflexia & $7(5.3)$ \\
\hline Total & $132(100)$ \\
\hline
\end{tabular}

$\mathrm{a}$ The most common clinical sign in neonates admitted for neonatal sepsis was respiratory distress in 51 cases (38.6\%).

sepsis signs and symptoms and positive blood culture) was $1545.65 \mathrm{pg} / \mathrm{mL}$ and in the second group (admitted with sepsis signs and symptoms and negative blood cul ture) was $14.79 \mathrm{pg} / \mathrm{mL}$, while in the control group, it was $11.04 \mathrm{pg} / \mathrm{mL}$ (Table 3). In this table, the most and the least values for maternal age at the time of delivery, gestational age, birth weight and the level of Il- 6 were included in three groups of this study.

The average levels of Il- 6 were 1545.65, 14.79 and 11.04 in groups A, B and control group, respectively.

Table 3. Mean of Variable Data in the Three Groups.

\begin{tabular}{|c|c|c|c|c|}
\hline Variable Data & $\begin{array}{l}\text { Group 1, mean }(S D),(n= \\
10),(\min -\max )\end{array}$ & $\begin{array}{l}\text { Group 2, mean }(\mathrm{SD}),(\mathrm{n}=10) \text {, } \\
(\min -\mathrm{max})\end{array}$ & $\begin{array}{l}\text { Group 3, mean }(S D),(n= \\
10),(\min -\max )\end{array}$ & Pvalue \\
\hline $\begin{array}{l}\text { Maternal age at the time } \\
\text { of delivery }\end{array}$ & $3.96(28.80)(22-34)$ & $6.10(32.90)(23-39)$ & $5.25(28.90)(20-36)$ & $0.001>$ \\
\hline Gestational age & $0.943(37-40)$ & $0.67 \pm(37.70)(37-39)$ & $0.67(38.30)(37-39)$ & 0.165 \\
\hline Birth weight & $3200(250)(2950-3450)$ & $3400(314)(3000-3900)$ & $3331(340)(2500-3500)$ & 0.186 \\
\hline The level of IL-6 & $1911(1545.65)(231-5000)$ & $17.89(14.79)(56-2.1)$ & $12.87(11.04)(44.5-2.63)$ & 0.587 \\
\hline
\end{tabular}

\section{Discussion}

Findings of this study necessitate to conduct further studies for confirming the role of interleukin- 6 in the diagnosis of early neonatal sepsis. Due to the high prevalence of the neonatal sepsis, the presence of a diagnostic marker for its early diagnosis is vital for the physician and the patient consequently. In a study which was conducted on 50 neonates suspected to sepsis at Isfahan University by determining a $30 \mathrm{pg} / \mathrm{mL}$ cut-off for this inflammatory marker, they concluded that the higher level is an important predictor of early neonatal sepsis (2).

In another study which was performed on 700 neonates suspected to neonatal sepsis at Shahed university by Bor- na et al. the subjects according to their clinical signs and laboratory results were divided into three groups and laboratory findings were compared in order to confirm the existence of neonatal sepsis. This study indicated that the CRP has a specific clinical sensitivity (79-85\%), but its positive predictive value is low in neonatal sepsis (36\%) (1). Another study was performed on about 760 neonates in Mashhad University of Medical Sciences by Mamoori et al. This study showed that other factors (neonatal Asphyxia, low APGAR score at birth) may also affect on the level of IL-6 in neonatal sepsis. Therefore, in this study, considering the level of IL-6 in prediction of neonatal infection, neonates with asphyxia or low APGAR score were excluded (3). 
The researchers found that the repeated evaluation of IL-6 after 12-24 hours isn't useful because it goes back to its initial level (3). In another study done on 309 premature neonates (24-31 weeks) by Dr. Alice Goop Firth, it was indicated that the levels of IL-6 are higher in preterm neonates and this increase is associated with some complications such as NEC PVL (abdominal Leukomalacia and inflammatory bowel necrosis). One of the most important features of this study is using the new technique of Electrochemiluminescence (ECL) compared with the most previous studies in which Enzyme-linked immunosorbent assay (ELISA) method has been used. Moreover, to obtain plasma level of interleukin- 6 unlike some previous studies in which the test depends on a test taker, and to obtain the exact level of plasma IL-6, a fully automated machine Elecsys 2010 was used which is not dependent on technicians (7-9). To achieve more precise and reliable probability of bacterial growth in blood cultures, Bactec culture medium in Becton Dickinson device was used rather than the common blood Agar culture, especially for neonates. Bactec culture medium has advantages compared with the other common microbial culture mediums such as its tangibility in case of the presence of any bacteria and in the previous studies, it had never been negative when other cultures had been positive (10).

Prevalence of definite sepsis in this study was $7 \%$ that is lower than that in developing countries because the premature neonates, neonates with birth asphyxia and previous admission were excluded from this study. In group $\mathrm{A}$, the most common etiologic bacterial agents detected in the Bactec blood culture medium were streptococcus group B (GBS) and S. epidermis (SE) that there were not differences between them, considering the prevalence of bacteria causing neonatal sepsis, in the other studies conducted in the country; the prevalence of gram-negative bacterial agents were relatively lower than that of the other etiologic bacterial agents (0.7\%).

The most prevalent clinical signs and symptoms leading to the admission of neonates were neonatal respiratory distress in 51 neonates (35.9\%). No significant correlation was observed between the levels of IL- 6 and type of symptoms leading to the admission of neonates. Between the two died neonates, one was identified with gram-positive bacteria in his blood culture and the level of IL- 6 was more than $5000 \mathrm{pg} / \mathrm{dL}$, and the other neonate was and diagnosed with Tachypnea while his blood culture was negative and the neonate level of IL-6 was $409 \mathrm{pg} / \mathrm{dL}$.

On the whole, a comparative test indicates that there is a significant difference between the levels of interleukin-6 in the three groups (P value $=0.001)$. This result not only omits all the probable confounding factors in plasma level of IL-6, but also emphasizes the role of this useful inflammatory marker in detection of neonatal sepsis.

\section{Acknowledgements}

There is no Acknowledgments.

\section{Authors' Contribution}

None declared.

\section{Financial Disclosure}

There is no Financial Disclosure.

\section{Funding/Support}

There is no Funding/Support

\section{References}

1. Adib M, Navaei F, SahebFosul F. Evaluation of interleukin-6 for early diagnosis of neonatal sepsis in comparison with crp. Med JEsfahan Univ Med Sic. 2008;82.

2. Boskabadi H, Maamouri G, Afshari JT, Ghayour-Mobarhan M, Shakeri MT. Serum interleukin 8 level as a diagnostic marker in late neonatal sepsis. Iran J Pediatr. 2010;20(1):41-7.

3. Mamouri gholamali M, Boskabadi H. Evaluation of Interleukin-6 for Early Diagnosis of Neonatal SEPSIS. Med J Mashhad Univ Med Sic. 2006;49(93):253-60.

4. Santana C, Guindeo MC, Gonzalez G, Garcia-Munoz F, Saavedra P, Domenech E. Cord blood levels of cytokines as predictors of early neonatal sepsis. Acta Paediatr. 2001;90(10):1176-81.

5. Verboon-Maciolek MA, Thijsen SF, Hemels MA, Menses M, van Loon AM, Krediet TG, et al. Inflammatory mediators for the diagnosis and treatment of sepsis in early infancy. Pediatr Res. 2006;59(3):457-61.

6. Kurt AN, Aygun AD, Godekmerdan A, Kurt A, Dogan Y, Yilmaz E. Serum IL-1beta, IL-6, IL-8, and TNF-alpha levels in early diagnosis and management of neonatal sepsis. Mediators Inflamm. 2007;2007:31397.

7. Guglielmo-Viret V, Attree O, Blanco-Gros V, Thullier P. Comparison of electrochemiluminescence assay and ELISA for the detection of Clostridium botulinum type B neurotoxin. J Immunol Methods. 2005;301(1-2):164-72.

8. Guglielmo-Viret V, Thullier P. Comparison of an electrochemiluminescence assay in plate format over a colorimetric ELISA for the detection of ricin B chain (RCA-B). J Immunol Methods. 2007;328(1-2):70-8.

9. Oh ES, Mielke MM, Rosenberg PB, Jain A, Fedarko NS, Lyketsos CG, et al. Comparison of conventional ELISA with electrochemiluminescence technology for detection of amyloid-beta in plasma. $J$ Alzheimers Dis. 2010;21(3):769-73.

10. Berger SA, Battat A, Halperin E, Stein J, Dan M. Comparison of an agar slide blood culture device with Bactec $6 \mathrm{~B}$ for the detection of bacteremia. Am J Clin Pathol. 1987;87(2):272-5. 